\title{
Study on College English Test (CET) Comprehensive Management Information System Based on Multi-system Data Integration
}

\author{
Qin Lele $^{1 *}$, Yin Shaoqing ${ }^{2}$ and Dong Ganghui ${ }^{3}$ \\ ${ }^{1}$ Hebei University of Science and Technology, Shijiangzhuang 050000,China; \\ ${ }^{2}$ Handan Polytechnic College,Handan 056000,China;3.Shijiazhuang Guojicheng \\ Primary School,Shijiazhuang 050000,China \\ Mr_qin@163.com \\ Abstract
}

\begin{abstract}
National College English Test (CET) is a nationwide examination that Ministry of Education is in charge of, with over 20 million exammees sitting CET every year. In recent years, on the basis of conventional written test, there are another three kinds of tests available, namely Internet-Based CET, Comparative CET and Predictive CET, which utilizes as many as five different sets of Software, with nodirect data sharing layer, for management. Therefore, the author succeded in devetopment of CET management information system based on multi-system data integration technology. This thesis puts forward corresponding solutions to problems, like indomplete functions and unfriendly data interface, occurring in the usefof original multiple system, focuses on introducing the methods for centralized integhation of basic data at the data sharing layer, realization of algorithm for intelligent arrangement of examination supervision and that for sampling of student qualification for COmparative CET, and realization of functions like standardized processing of scores in above-mentioned three tests, and elaborates the thinking clews for CET management in some university by combining with the said system. The said system has been put intorservice successfully, with good value for popularization.
\end{abstract}

Keywords. CET; system development; data integration

\section{Introduction}

National College English Test (CET) is a nationwide examination that Ministry of Education (MOE) is in charge of and Department of Higher Education of MOE hosts. Being divided into CET-4 and CET-6, CET has been put into practice for over 20 years since 1987, as one of examinations with the largest scale and the largest influence nationvide, and there are over 20 million examinees sitting CET every year. In recent years, CET Committee has developed Internet-Based CET (IBCET) by utilizing the advantages of modern information technology. IBCET can test the examinees' ability to comprehend English audio and video and express in oral English in a comprehensive way, grant the certificate equivalent to traditional CET certificate and offer four opportunities for examinees to take the examination every year. In order to control the difficulty of test questions in future more effectively and accordingly report the examinees' final scores more scientifically, CET Committee has developed Comparative CET and Predictive CET in a few pilot schools, that is to sample from examinees attending the current test, ask them to complete Comparative CET and Predictive CET about one week ahead of the official test and compare their scores with the counterparts achieved in the official test, in a bid to adjust the difficulty of future test questions. To sum up, CET can be divided into the following forms: (1) conventional CET; (2) IBCET; and (3) Comparative CET and Predictive CET. Varying from the forms of implementation and the management information system used, the final scores of these three tests are recognized by each other 
and equivalent mutually and examinee samples for Comparative CET and Predictive CET come from the official test. Therefore, it is necessary to design a CET management system on the basis of multi-system data integration, so as to realize the data resource sharing and improve the organization efficiency and level of CET.

\section{Status Quo of Research}

Based on the examination management under the credit-system teaching management mode of higher education schools, Li Xin and Liang Changyi developed examination management information system and proposed new views on the examination arrangement in 2012. At the same year, Hu Xinyu designed an automatic exam-arranging arithmetic according to the graph theory, which can solve problems effectively and receive better results. In 2005, aiming at the special requirements on the seating arrangement of a multidisciplinary and large-scale examination, Zou Runqi and Du Liping put forward a new arithmetic and analyze its correctness and space complexity. The same year, Wang Zhuping and Chen Xiang put forward the realization methøds of key modules based on the general design plan of exam management system by conpuler application. Up to now, the researches and reports on large-scale exam management system are quite few. There is still a blank for the research on data integration technology of multimanagement system for Category A examination.

\section{Realization and Overall Design of System Data Resource Sharing Layer}

\subsection{Realization of Data Resource Sharing Layer}

For the sake of managing these three tests as a whole more efficiently, what comes first is to complete the data integration and sharing, in this way, it is essential to find necessary sharing points in three different data eategories, as shown in Figure 1, with the forms of integration as follows:

(1) Examinee information for $\mathrm{BBCET}$ and convention CET comes from the category of examinee rollata, while the reyiew of CET-6 examinee qualification does from the category of examinee scores,

(2) Examinee information for Comparative CET and Predictive CET comes from that in the category of congentional CET data and are available for the examinee qualification is reviewed on the occasion of enrollment for conventional CET-6.

(3) Final scores of three different tests are equivalent and thus are all submitted to the category of examinee scores.

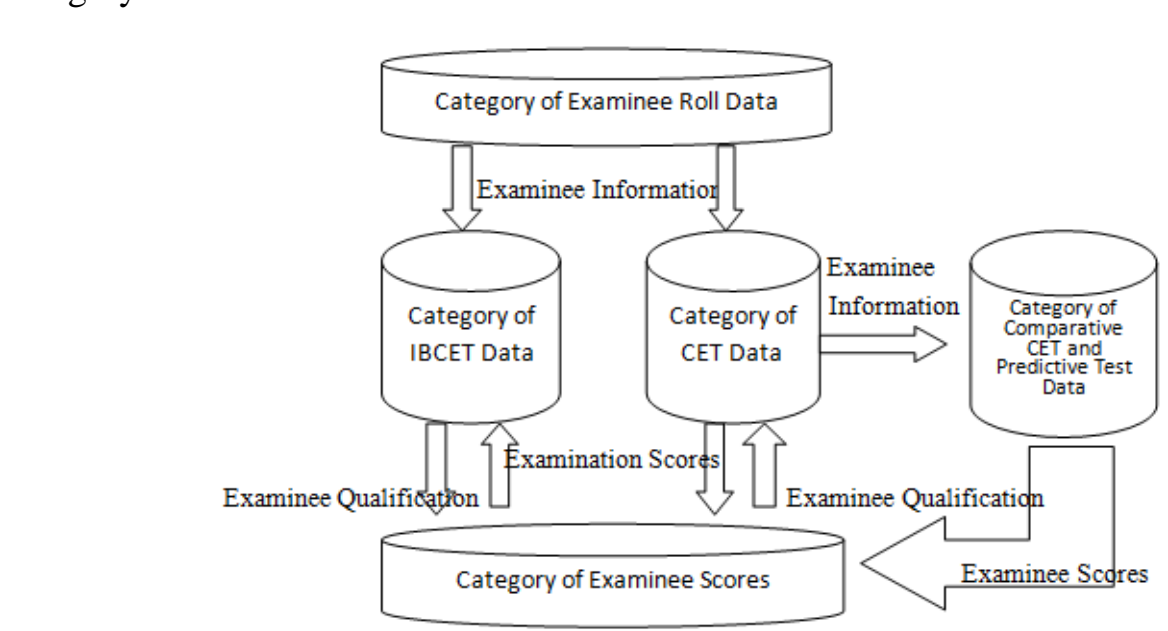

Figure 1. Realization of Data Resource Sharing Layers in 3 Tests 


\subsection{Overall Design of System}

The structural diagram of system function is as shown in Figure 2:

(1) System Setting: Examination batch setting, data backup, data compression and basic data management;

(2) Conventional CET Management: Import of examinee qualification database, maintenance of standard examinee information, printing of examinee enrollment information form, export of sample data, export of enrollment system data, acquisition of examination room arrangement, authentication of examinee qualification, maintenance of examination supervisor data, export of online examination supervisor and enrollment data, printing of examination materials, designated and random arrangement of examination supervisors and so on;

(3) IBCET Management: Import of examinee qualification database, maintenance of standard examinee information, export of standard examinee roll data, export of standard examinee enrollment information data, and so on;

(4) Comparative CET Management: Import of examineg dagtabase, randon examinee sampling, examination room arrangement, and so on;

(5) Score Management: Standard processing of scores in three tests, score filing, score inquiry, score analysis and statistics etc.

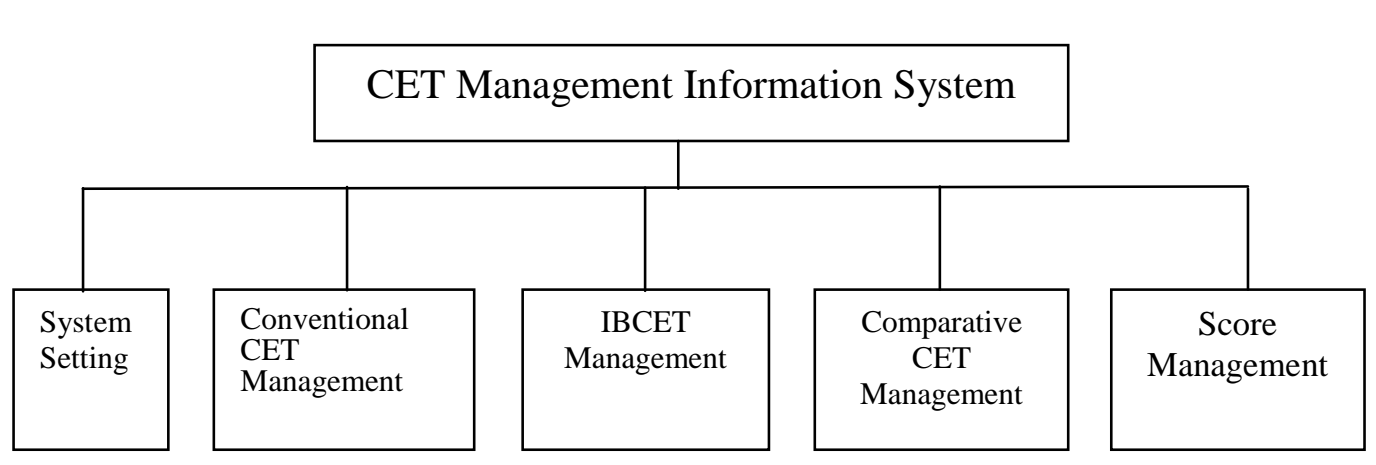

Figure 2. Structural Diagram of CET Management System Functions

\subsection{Design of Data Integration}

Conventional methods for data integration can usually be divided into pattern integration and datareproduction, with the former not realizing data exchange among data sources and the latter generally processing large delay, both deficient in real-time consistency of data. This research adopts the method named "fragmentation, reorganization and conception pattern" for data integration as follows: 1 . To fragment the data attribute set of data resource to be integrated; 2 . to organize the concept database by adopting the existing standardization system or establishing models of corresponding sectors according to the top-level design thought; 3 . to organize multiple theme databases on the basis of concept database and utilize the theme databases to support the development respective business systems.

\section{Design of Conventional CET Management Subsystem}

For the sake of further better management, National Education Examinations Authority (NEEA) of MOE developed National College English Test Roll Management System (the Examination Affairs System) for the purpose of examination organization work in 2005. As Hebei is a major province of education, with approximately two million examinees taking CET every year, in order to adapt to new situations, Hebei Education and Examination Academy (HBEEA) developed National College English Test Online Enrollment and Payment System (the Enrollment System) for the purpose of examinee enrollment in 2011. 


\subsection{Introduction to functions of the Examination Affairs System and the Enrollment System}

4.1.1 Basic functions of the Examination Affairs System: The Examination Affairs System is developed under the organization of MOE. From 2005 to 2011, schools were responsible for entering basic information, like school information and department information, and examinee-related data via the said system, submitting the enrollment data exported from the said system to the superior, receiving the examinee arrangement data from the superior before the examination, importing into the said system and printing the admission tickets and the lists of examination rooms. Therefore, the said system basically covers the entire examination management process, with basic functions as follows:

1) Basic data management: Setting of initial language, basic information of school, that of campus, that of college, that of major and that of class etc;

2) Enrollment management: Examinee information management matching of examinee and campus and matching of examinee and enrollment type etc.

3) Examination room arrangement management: Exanlination room arrangement, printing of admission ticket and printing of other examination matérials elc.

4) In-examination management, post-examinat on management, score management and so on

4.1.2 Basic functions of the Enrollment System? The Enrollment System is developed under the organization of HBEEA, in B/S computing mode. In this system, examinees conduct online information declaration and payment confirmation through WEB end, with individual as unit; examinee name, gender, ID No. and examination type are uploaded in batch by the schpols where examinees are, as the examinee qualification database; in addition, before examinee enrollment, schools must well maintain data like school, department, major and class in the Examination Affairs System, export in terms of package and upload to the Enrollment System for examinees to select when they enroll. The Enrollment System contains main functions as follows:

1) Basic data management Codes and values of information in this section come from the Examination Atfairs System,

2) Examineequalification database management: Schools provides related information, including: name, gender ID No. and examination type, which is the basis for examinees to $\log$ in the system for enrollment.

3) Enrollment Management: Examinees in the examinee qualification database log in the website with name, gender and ID No. for enrollment and payment.

\subsection{Major Problems}

1) Sine lots of basic information in the Enrollment System comes from the Examination Affairs System, data maintenance in the Examination Affairs System seems very important; however, the settings of class and major are required to update every year while such data need to be imported one by one in the Examination Affairs System, subject to errors, and are not allowed to change once being entered into the Enrollment System;

2) In the examinee enrollment qualification database, there are only four fields namely name, gender, ID No. and examination type and the rest allows examinees to select in the Enrollment System by themselves, which often leads to campus, college, class and similar information selection mistake; according to incomplete statistics, the mistake rate of examinee information in the school where the author is exceeds $6 \%$, causing inconvenient to the examination organization work; 
3) Functions in the Examination Affairs System are defect, e.g. without random examination supervisor arrangement function or campus examination timetabling function;

4) The information sharing layers in several systems are not combined in a friendly manner, e.g. the Examinee Roll System exports the examinee roll information in terms of EXCEL while what the Enrollment System requires is such information in terms of FOXPRO.

Therefore, in combination with routine examination management, it is of great importance to build a new system among several systems.

\subsection{Analysis on Flow of Multi-system-based CET Management System}

According to the foregoing problems, it is necessary to set up the multi-system-based CET management system (the Management System) as the intermediate layer aanong the Examinee Roll System, the Examination Affairs System and the Enrollment system, in a bid to realize the friendly sharing of multiple system data layers and the "individualized" management function, with the flow as follows:

(1) The Management System acquires the examinee roll information from the school general affairs system to complete the examination qualification database;

(2) Examinee sample data (including information of college, major, grade and class) are exported by means of SQL sentence and imported into the Examination Affairs System to complete the addition of basic data,

(3) Basic data and the examinee qualification data are exported from the Management System and the Examination Affairs \$ystem with related functions and imported into the Enrollment System;

(4) The examinee information form is printed via the Management System and delivered to examinees, examinees complete related information in the Enrollment System according to related data on the information form and any incompliance with requirements will lead to disqualification for examination;

(5) HBEEA dístribytes the examinee data, imports into the Examination Affairs System and the Management System, reviews whether the examinee information for examination is completed correctly or not, and prints the admission tickets via the Examination Affairs Systeni:

(6) The online database is exported via the Management System and allows the examination supervisors to enroll at $\mathrm{B} / \mathrm{S}$ end;

(7) The examination supervisor enrollment data are downloaded to the client end of the Management System;

(8) Examiration supervisors are arranged according to set algorithms and management modes;

(9) Re)ated materials are printed via the Management System.

See Figure 3 for the data flow chart. 


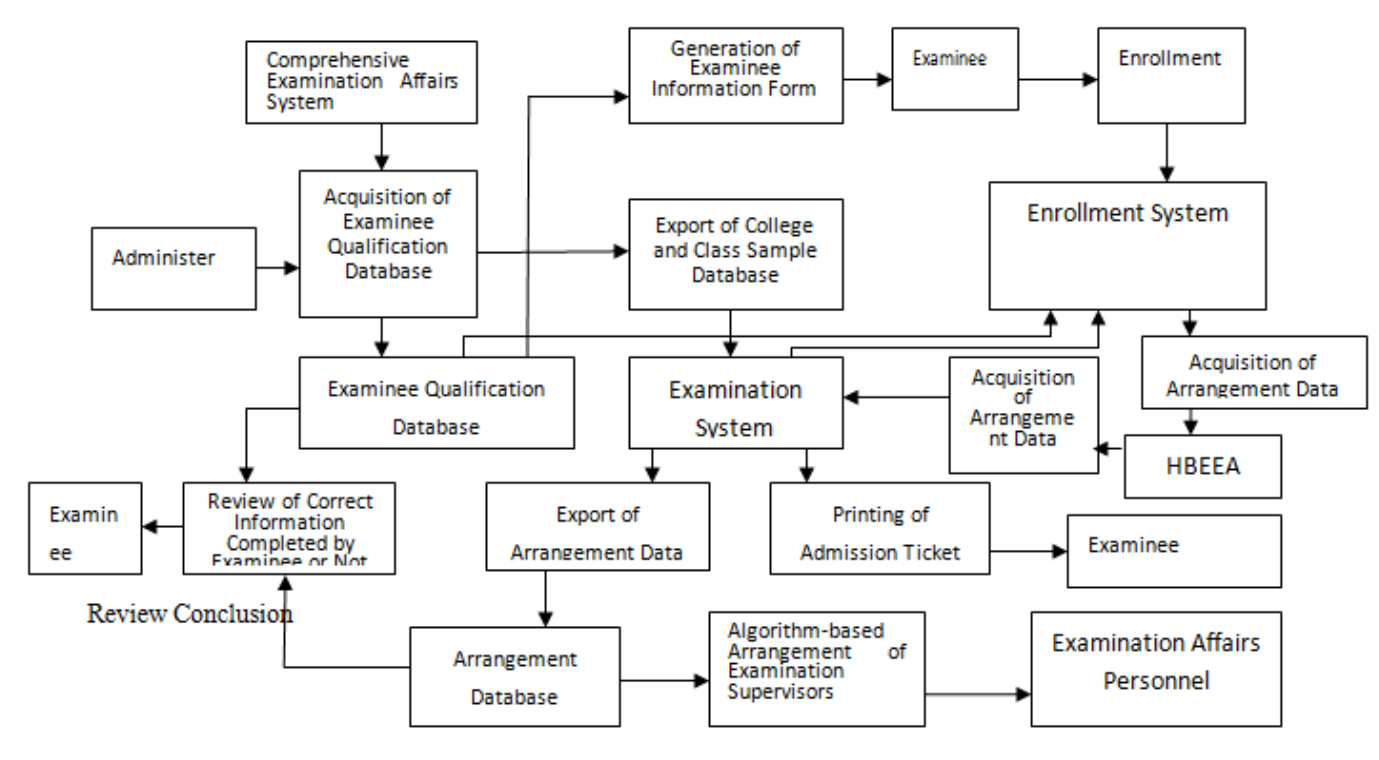

Figure 3. Flow Chart of Multi-system-based CET Management System

\subsection{Design of Main System Functions and Realization of Algorithms}

\subsubsection{Realization of Inter-system Basic Data Import and Export Function}

The basic data shared by the Enrollment System and the Examination Affairs System include: the school code table and the campus code table etc., as shown in Figure 4.

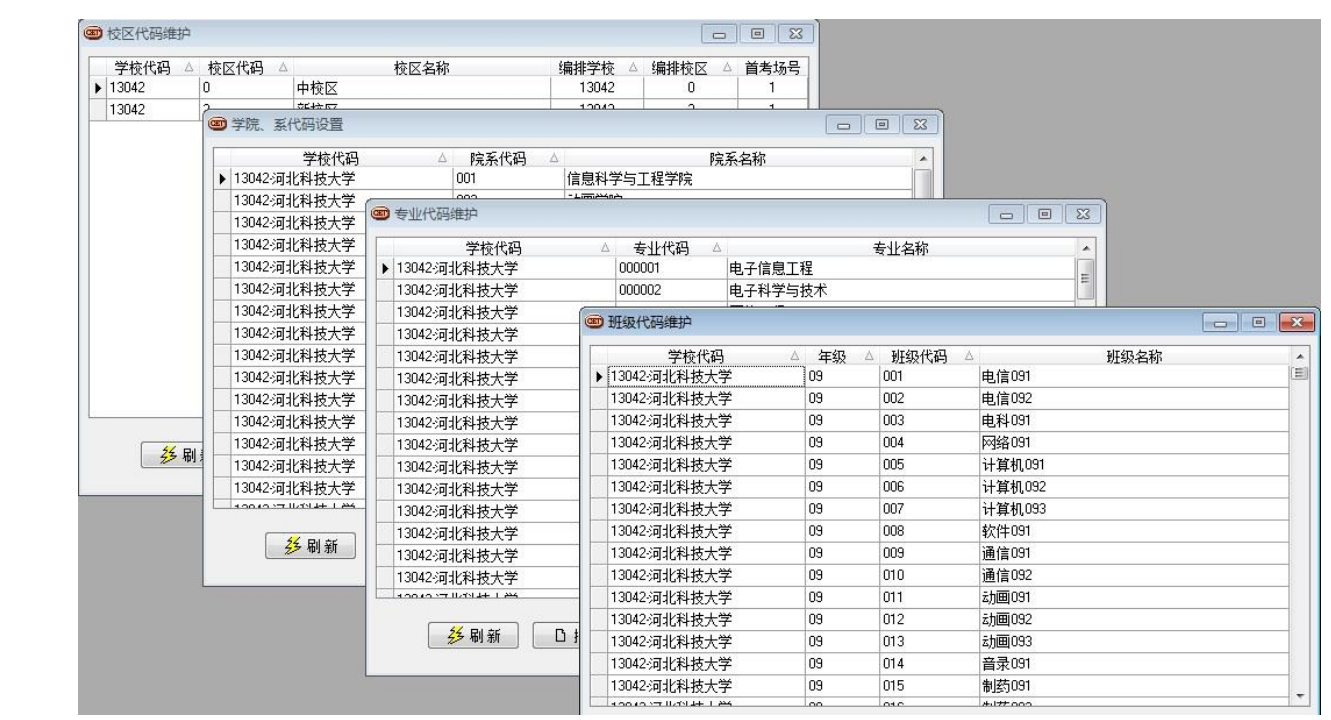

Figure 4. Basic Data Maintenance Interface in Examination Affairs System

It can be seen that, many codes need to be imported in the Examination Affairs System one by one, without the function of data import. The entry work is complicated and easy to make mistakes; if something is wrong with basic data, examinees will encounter the enrollment information error or be unable to select correct related information in online enrollment. The practice in this research is, to import the roll information of examinees qualified for the examination into the Management System so that there would be corresponding college, class and major information in the Management System, then export all emerged college, class and major information to the temporary form by utilizing the "distinct" function in SQL sentence, supplement other corresponding codes 
and next complete the addition of basic data in the Examination Affairs System, as shown in Figure 5.

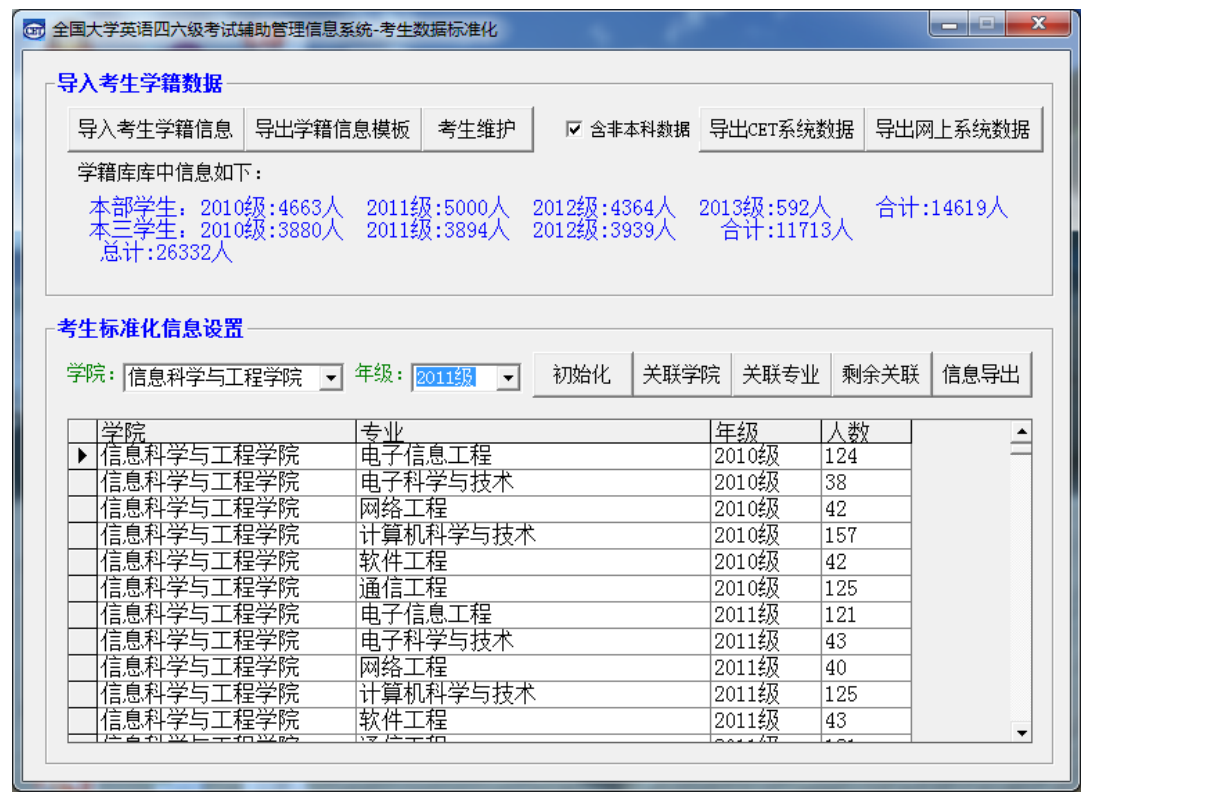

Figure 5. CET Management System Sample Data Setting and Export Interface

\subsubsection{Realization of Exammee Qualification Review Function}

In Hebei, many schools have more than 15,000 examinees in separate campuses and accordingly it is necessary to designate the examination campuses for examinees. The practice of the system is to set a campus field when importing the examinee qualification database, enter related information in the said field, maintain in the system, print "Examinee Information Form" for every examinee before enrollment and ask examinees to conduct the online information completion according to the requirements of the form, with any non-conformits sybject to corresponding settlement.

$\Omega$

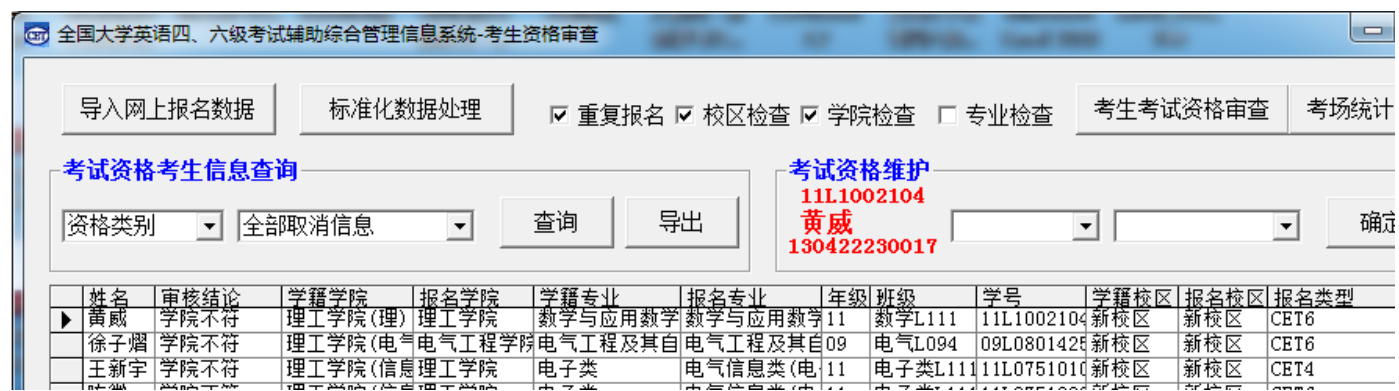

Figure 6. Examinee Qualification Review Interface

\subsubsection{Examination Supervisor Enrollment Management and Realization of Corresponding Arrangement Algorithm}

Take the school where the author is for example. There are approximately 12,000 CET4 examinees in the new campus as examination site, involving 400-odd examination rooms and seven teaching buildings. According to related examination regulations, the arrangement of examination supervisors must be released within two hours ahead of examination. Simple random arrangement of examination supervisors and centralized 
announcement of arrangement results before the examination would lead to the following problems:

(1) There would be more than 800 examination supervisors concerned and it would be very difficult for them to find their information if the arrangement results were released in a centralized manner;

(2) It would take a long time for examination supervisors to reach the designated examination rooms due to long distances between buildings even if they succeeded in finding their information.

On the basis of not violating the related examination regulations, the system processes as follows: (1) Setting random numbers for chief and deputy examination supervisors; (2) Calculating the quantity of examination rooms in every teaching building; (3) Allocating examination supervisors to respective teaching buildings according to random numbers; (4) Announcing exanimation supervisors corresponding to every teaching building in the afternoon before examination; (4) Setting random numbers for chief and deputy examination supervisors once again; (5) Allocating examination supervisors in every teaching building to respective examination rooms according to random-numbers; (6) Announcing the specific arrangement of examination supervjsors at respective teaching buildings within two hours ahead of examination. In thrs way, the foregoing problems would be settled, as shown in Figure 7.

Furthermore, for CET in the first half of year, besides normal English test, there are minority languages like French, Japanese, German and R đussian. The examination rooms for minority language tests are numbered, behind English test. Even if examinees are less, special personnel are also required for management, hence, special personnel must be designated to minority language examination cooms. On the basis of not violating the random arrangement of examination supervisors tor English test, the system adopts an algorithm, in which the randolm number of examination supervisors in common examination rooms ranges between 100000-900000 while that in designated examination rooms between 200000-290000, e.g. 231369, with 2 standing for designated arrangement, 3 for new campus, $\wedge 1$ for CÉT-4 and 369 for examination room number. In arrangement, what first set is the information of examination rooms for designated personnel and then the system completes the number of building and that of examination room where designated personnel superyise the examination, with the rest examination rooms subject to random arrangement.

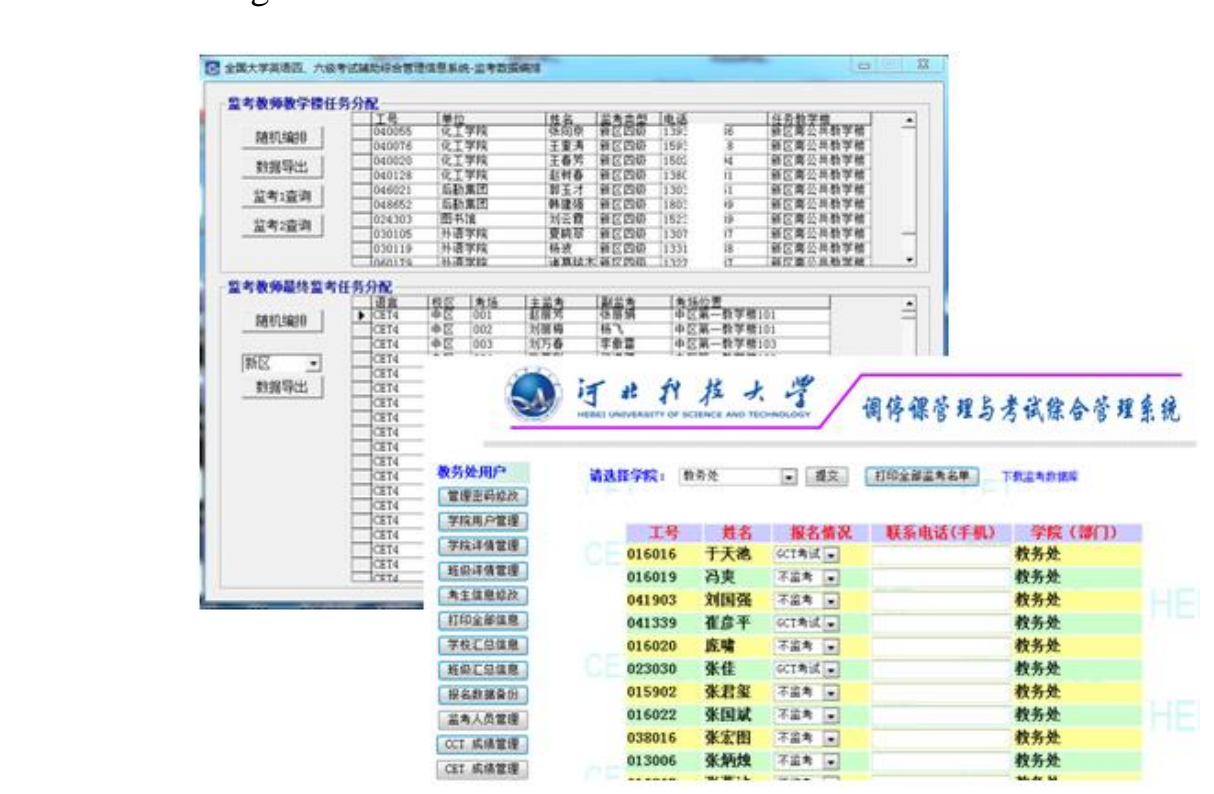

Figure 7. Examination Supervisor Random Arrangement Interface and Online Enrollment Management Interface 


\section{Design of Comparative CET and Predictive CET Management Subsystems}

Comparative CET and Predictive CET (Comparative Tests) are a standard measure for future examination questions, that is to sample from examinees attending the current test, ask them to complete Comparative CET and Predictive CET about one week ahead of the official test and compare their scores with the counterparts achieved in the official test, in a bid to adjust the difficulty of future test questions and the coefficient of scores for admission. Therefore, the basic design functions of this system are: randomly sampling data from examinees for official test, randomly arranging to tests of different types, accordingly further completing examination room arrangement, admission ticket printing and score filing etc.. See Figure 8 for the data flow chart and the main system interface.

\subsection{Data Sampling}

Sample data shall be representative, which is the most important feature. For this reason, this system adopts the following measures in design: (1) to set up the sample database by sampling examinees attending CET for the first time in combination with the historical score database; (2) to pool examinees in several colleges for management convenience; (3) to set the random number and anange in the ascending sequence; (4) to set the quantity of examinees in every colleges in proportion; (5) to sample certain quantity of examinees taking the official test according to the standard for quantity of examinees taking Comparative CET and Predictive CETy and (6) to arrange examinees into different examination types randonify

\subsection{Score Filing}

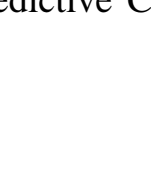

Scores delivered by HBEEA are in the terphs of text file, containing admission ticket numbers and scores of examihees, thus it is necessary to link the examinee roll database, the database of examinees taking the official test and the score text file and enter into the historical score database according to standard format.

\section{Design of Internet-Based CET Management Subsystem}

CET reform is being advanced constantly, including two sections: reform in examination contents and in examination methods; the former is to advance the computer examination by cint of information technology and the latter is to intensify the examination of listening and oral English. Therefore, Internet-Based CET (IBCET) has been implemented since April, 2009, with scores equivalent to those achieved in conventional CET. Accordingly, the management process can be brought under this system and scores can be managed in a centralized manner in the historical score database. See Figure 9 for the data flow chart and main interface of subsystem.

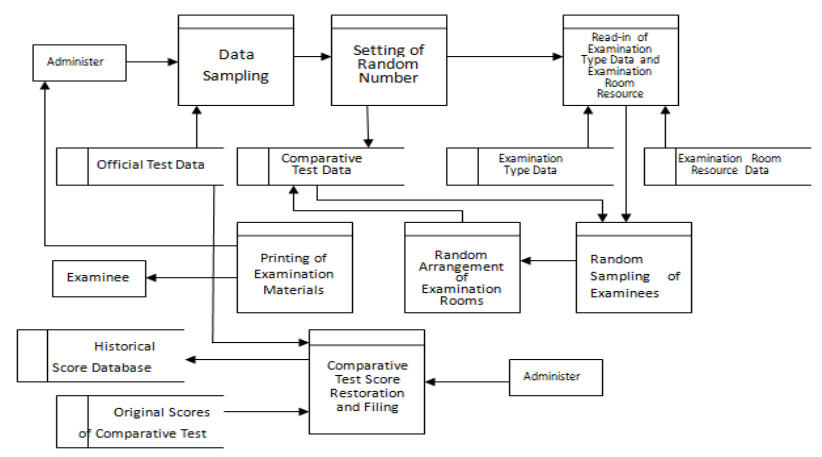

Figure 8. Data Flow Chart of Comparative CET Management Subsystem 


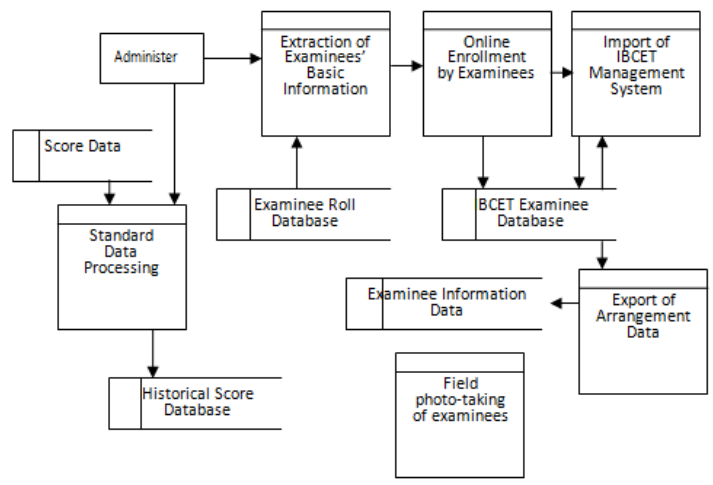

Figure 9. Data Flow Chart of IBCET Management Subsystem

\section{Conclusion}

It took three years to develop the said system, with which the management of CET, IBCET, Comparative CET and Predictive CET in Hebei University of Science and Technology has been done for six times, and the data integration of four systems for three tests and the centralized management of score databases been realized. The said system is stable and reliable, with all indicators meeting design requirements, greatly improves the work efficiency and the management level and enables the examination affairs management work of CET to be modernized, highly-efficlent, accurate and intelligent. The realization of the said system is of significant significance in application and this is a well system worthy of popularization.

\section{Acknowledgement}

This work is financidily supponed by College and University Scientific and Technological Reseånch Program of Hebei Province: Study on College English Test (CET) Comprehensive Management Information System Based on Multi-system Data Integration (QN20131139). The authors are grateful for their support.

\section{References}

[1] Q. Lele and Z. Jinfeng, "Development of Dangerous Source's Monitoring and Management and Emergency Rescue Decision Making Support Information System”, Journal of Computers, vol. 6, no. 4, (2011), pp. 732-7,39.

[2] S. C. Mista and A. Mondal, "Identification of a company's suitability for the adoption of cloud computing and modeling its corresponding Return on Investment", Mathematiealand Comouter Modelling, vol. 6, no. 2, (2011), pp. 504-521.

[3] Q. Lele, Z. Huixiao and Z. Jinfeng, "The application of RFID in logistics information system", Proceedings of 2008 IEEE International Conference on Service Operations and Logistics and Informatics, vol. 1, no. 1, (2008), pp. 534-537.

[4] Q. Lele, H. Tao and Z. Huixiao, "Development of archives management information system based on .NET multi -tier architecture", Proceedings 2009 3rd IEEE International Symposium on Microwave, Antenna, Propagation and EMC Technologies for Wireless Communications, vol. 1, no. 2, (2009), pp. 1234-1237.

[5] D. yixin, H. xun and C. Jingjing, "Research on Implementation Method of Data Integration Projects based on SFRAC", Electric Power Information Technology, vol. 11, no. 1, (2013), pp. 66-70.

[6] G. Dan, "Data Integration Technology Research Based on ORM", Value Engineering, vol. 12, no. 1, (2012), pp. 194-195.

[7] Q. Lele and Z. Xin, "Design and Realization of Information Service Platform of Logistics Parks Based on Cloud Computing", AISS, vol. 4, no. 23, (2012), pp. 112-120.

[8] W. Xiuxia and Q. Bo, "It Began with SOA for Data Integration of System of Petrochina's Archives Management", Computer Knowledge and Technology, vol. 7, no. 15, (2011), pp. 3582-3584. 


\section{Author}

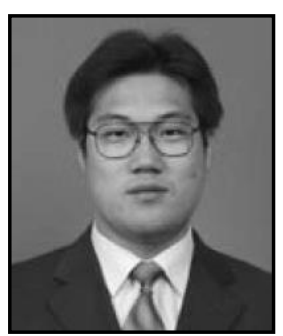

Qin Lele, male, was born in Zhangjiakou, Hebei, China on March 19, 1978. He earned a Master Degree of Engineering from Nanjing University of Science and Technology, China in 2006.His major field of study is the analysis and design of management information system.

He has been in the research of management information system, and is now dean of Teaching Operation Management, Office of Teaching Affairs in Hebei Univesity of Science and Technology. He has been teaching more than 10 courses at various levels including Management Information System, MIS Curriculum Design, and Database Principle. He has published "A Case of Environmental Engineering CAD Application" (Beijing, China: Chemica Industry Press, 2005) , "A Case of Water Treatment Engineering CAD Application" (Beijing,China: Chemtcal Industry Press, 2002) . He has published more than 20 articles, 15 of which were indexed by EI.His research interests are the analysis and development of MIS.

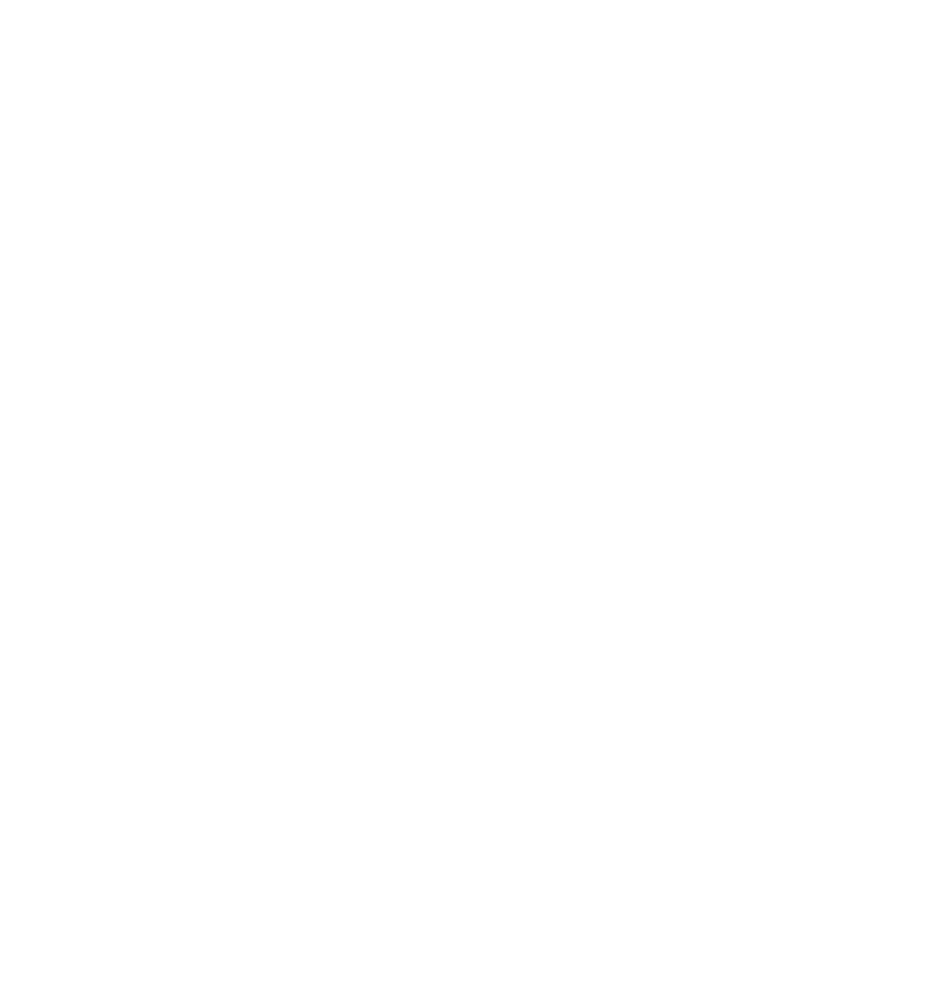


International Journal of Multimedia and Ubiquitous Engineering

Vol.9, No.8 (2014)

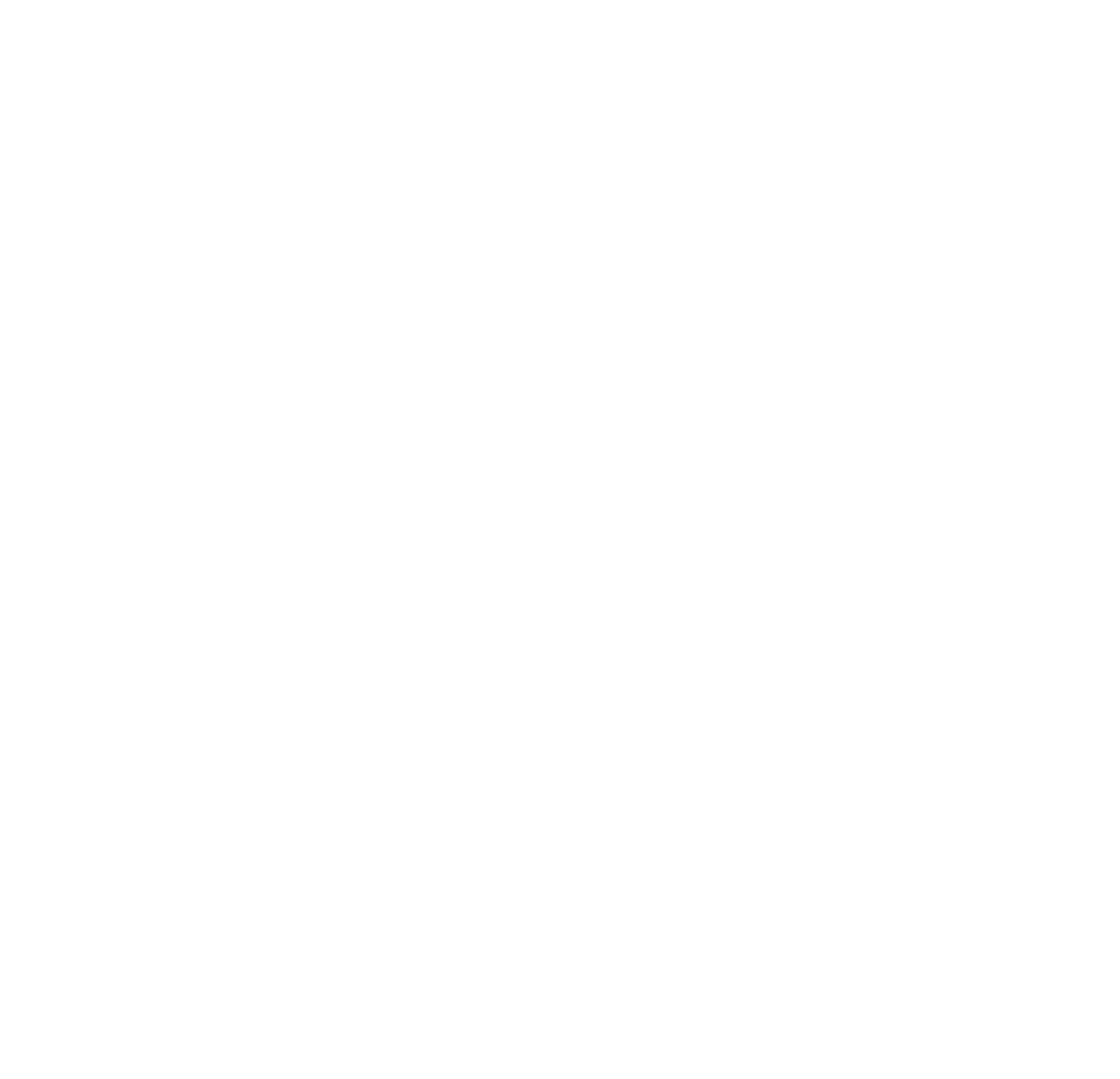

